\title{
Développer les capacités de reformulation chez les maîtres de l'école bilingue en contexte subsaharien
}

\author{
Colette NOYAU \\ UMR 7114 CNRS MoDyCo \\ Université Paris Ouest Nanterre La Défense \\ cnoyau@,u-paris10.fr \\ http://colette.noyau.free.fr
}

\section{Usages de la reformulation chez les maîtres : le problème didactique}

Dans la multitude d'écrits sur les difficultés des systèmes scolaires subsahariens et les façons de faire progresser l'école dans ces contextes plurilingues, l'un des facteurs évoqués est la faiblesse de la formation des maitres, tant pédagogique que dans la maitrise de la langue d'enseignement (cf. Garry et al. 2008 ; Karsenti et al. 2007).

On a identifié un aspect des comportements langagiers des maitres en classe qui se situe à la croisée de ces deux aspects : la tendance à ne pas faire usage de la reformulation. Cette tendance peut s'expliquer, en partie, par les limitations du répertoire linguistique des maîtres, mais elle relève aussi d'une conception des connaissances scolaires comme devant se formuler d'une unique façon, celle du manuel et des questions d'examen. Et il ne s'agit pas tant selon nous d'un phénomène d'insécurité linguistique (Bretegnier \& Ledegen, 2002) que d'une conception de l'éducation scolaire comme reposant sur la mise en mémoire de la parole exacte qui s'impose à tous, une tendance à la 'littéralité' des textes de savoir. Les reformulations sont alors vues comme des étapes nécessaires mais à dépasser pour aboutir à la formulation stabilisée seule souhaitée, qui est à mettre en mémoire (Noyau, 2002a, 2003).

Ces pratiques ont des conséquences non négligeables en ce qui concerne les apprentissages chez les enfants, tant pour l'appropriation de la langue, qui suppose la capacité de faire varier les formulations en fonction des contextes, des points de vue et des effets pragmatiques, que pour celle des connaissances en jeu, car la bifocalisation sur la langue et les contenus véhiculés simultanément (Bange, 1992) constitue une situation cognitivement conflictuelle, qui souvent se résout dans les systèmes scolaires subsahariens étudiés au détriment des connaissances à construire (Noyau, 2004, 2007 ; Noyau \&Vellard, 2004). Or dans les conditions de l'école francophone en contexte plurilingue, l'exposition à la langue française est restreinte hors de la classe, et c'est la langue des maîtres qui constitue de fait le français de référence pour les élèves (Noyau, 2006a). Notons au passage que c'est le problème inverse qui est thématisé dans les situations canadiennes de scolarisation en L2 par immersion, où les élèves développent de bonnes capacités de communication orale dans la L2, en réception comme en production, mais où la fluidité est vue comme obtenue au détriment de la précision de la forme, notamment écrite (voir Lyster 2002).

Ces comportements des maîtres sont liés par ailleurs aux conceptions implicites des relations oral - écrit à l'école où l'oral est toujours tourné vers l'écrit (dérivé de --, ou allant vers --), étrange paradoxe dans des sociétés fondées sur l'oralité : dans la plupart des pays de notre étude, l'enseignement scolaire est unilingue, fondé exclusivement sur le français et nécessairement ancré sur l'oral, compte tenu du manque de matériel imprimé, mais un oral tourné vers l'écrit à différents titres. La langue française s'y présente essentiellement comme une "langue-à-écrire", et l'entraînement à un système de communication orale est marginalisé. Dans ces conditions, le double lien: acquérir la langue pour acquérir des connaissances et acquérir des connaissances dans une langue à découvrir, place l'élève devant une tâche cognitive très ardue (Noyau, 2004).

Rappelons aussi qu'en contexte hexagonal d'enseignement du français comme langue maternelle, on a pu montrer que la pratique des reformulations s'accroît avec l'expérience de l'enseignant (Garcia-Debanc \& Volteau, 2007 ; Volteau \& Garcia-Debanc, 2008, 2009).

Nous allons nous focaliser pour le présent travail sur les reformulations à l'oral entre maîtres et élèves en 
classe au primaire à l'école subsaharienne, dans les systèmes dits 'classiques', où seul le français a droit de cité, et qui ignorent la situation de plurilinguisme du milieu. Nous montrerons qu'en fait, ce n'est pas tant le manque de reformulations qui est en cause, que le profil des activités de reformulation, du point de vue de leurs types descriptifs, mais aussi du point de vue de leurs fonctions, et de leur insertion dans un parcours cognitif de construction de savoirs langagiers et disciplinaires - ce qui conduira à des propositions concernant la formation initiale et continue des maîtres.

\section{Formes et fonctions de la reformulation : les appuis théoriques}

\subsection{Analyse des reformulations dans les travaux de didactique}

En didactique de la langue première (L1) comme en didactique d'une langue seconde (L2) de scolarisation, et dans la didactique des disciplines dites non linguistiques (DNL) (cf. Gajo 2007), on dispose déjà d'un ensemble de travaux qui analysent la reformulation, ses fonctions, et ses pratiques de la part des élèves et des maitres en classe. ${ }^{1}$ Mentionnons seulement, par exemple, les travaux canadiens de Lyster $(2002,2009)$ sur les fonctions de la reformulation dans les programmes d'immersion canadiens, où l'apprentissage de la L2 se fait par l'enseignement scolaire effectué totalement en L2, entre moments de centration sur le contenu et de focalisation sur la forme. En didactique du français LM, évoquons ceux de Garcia-Debanc et Volteau (2007 et 2009), qui tiennent compte aussi du rôle des formulations successives des EE entre eux et en interaction avec le $\mathrm{M}$ dans le processus de co-construction des savoirs.

Ce dernier aspect est par ailleurs étudié, à l'écrit, par Boré (2000) et Elalouf (2005), à travers les reformulations successives par les EE des brouillons vers un état de texte visé, en tant que processus visant à « écrire pour apprendre », sous l'angle des écrits intermédiaires et des relations reformulatives entre écrits successifs.

Quelques travaux analysent d'un point de vue acquisitionnel les reformulations chez des apprenants de différents paliers de scolarisation dans un contexte de francophonie diglossique, pour ce qui est du lexique des prédicats verbaux (Bedou-Jondoh \& Noyau, 2003 ; Kihlstedt, 2005 ; Noyau, 2003, 2005, 2008), à partir d'activités de restitution de récits entendus, dans des conditions semi-expérimentales. On a comparé les reformulations d'un conte par les apprenants après son écoute (restitution immédiate ou différée) au conte écouté. De ces études, relevons quelques résultats pertinents pour notre objet.

Ainsi, Bedou-Jondoh \& Noyau (2003), qui comparent systématiquement les types de reformulations par rapport au texte source, tant en L1 qu'en français (la tâche ayant été donnée dans les deux langues, gengbe et français), montrent que les apprenants les plus jeunes ( 8 ans, 3e année de scolarisation en français) se distinguent par leur recours à des verbes de base ${ }^{\mathrm{iii}}$ absents du texte, alors que les apprenants plus âgés (10 ans, 5e année de scolarisation en français) préfèrent la reprise à l'identique des verbes du texte source. Contrairement à certaines attentes qui reposent sur l'idée qu'il est plus facile de répéter que de varier, c'est avec la consolidation de l'acquisition que s'accroit la capacité de reprise à l'identique d'un message antérieur. En effet, il faut garder à l'esprit que l'écoute d'un texte suivie de sa reformulation constitue un double travail cognitif de compréhension suivie de reconceptualisation pour une nouvelle mise en mots. Sur ces mêmes données concernant le français, Noyau (2005) met en évidence le recours massif à un verbe peu fréquent en français, absent du texte source : puiser. Outre le biais thématique dû à ce que le fait d' «aller chercher de l'eau à la rivière » est une action clé du récit en question («Les deux amis ») ${ }^{\text {iii }}$, le corrélat du verbe puiser dans les parlers gbe de la zone (éwé[gbe], gen[gbe]) est hyper-fréquent pour des raisons d'environnement écologique, et passible d'emplois abstraits et phraséologiques. Ce sur-emploi spontané constitue un contre-exemple très visible à la tendance des apprenants à recourir aux verbes de base, qui renvoie à une reconceptualisation influencée par la L1.

L'étude de Kihlstedt (2005), quant à elle, compare des groupes d'apprenants de CM2 (11 ans, 6e année de scolarisation en français) et de $5 \mathrm{e}$ (13 ans, 8e année de scolarisation) dans le conte traditionnel de «L'enfant terrible». Certains verbes absents du texte source sont massivement employés dans les restitutions des apprenants : ainsi, aller et refuser. Le premier est un verbe de base qui se substitue 
commodément aux verbes de déplacement plus diversifiés du texte source, les déplacements du protagonistes jouant un rôle structurant dans ce récit. Le second est un verbe hyper-fréquent en français du Togo, par influence probable des parlers gbe du sud-Togo. Cette influence typologique de la L1 sur la lexicalisation des procès se fait sentir plus fortement chez les apprenants plus jeunes du français L2. Cet effet est par ailleurs davantage observable dans d'autres types de productions narratives, à partir de supports non-verbaux, où il n'est pas alors question de reformulation, mais simplement de formulation (cf. Noyau, 2002b).

Tous ces résultats nous rappellent que la reformulation est d'abord contrainte par le répertoire linguistique que possède le locuteur, et par sa disponibilité lors de la production - tant lorsqu'il s'agit de converger avec une formulation antérieure de soi-même ou d'autrui, que lorsqu'on souhaite s'en écarter. Les contours du répertoire linguistique des élèves se laissent deviner en analysant leurs reformulations en classe.

\subsection{La reformulation et ses analyses linguistiques}

Du point de vue linguistique, la reformulation a été particulièrement étudiée dans des travaux d'orientation interactionniste (reformulation par l'autre / par soi, initiée par soi-même / par l'autre, à fonction interactionnelle), depuis les années 70 (Gülich \& Kotschi, 1987; De Gaulmyn, 1987). Le linguiste recherche alors des marqueurs de reformulation qui signalent la relation entre formulations -marqueurs qui ne sont ni nécessaires ni suffisants, car ils se bornent parfois à un indice prosodique peu fiable, ou sont absents lors des reformulations à distance.

Un sous-ensemble des reformulations constituent des paraphrases linguistiques qui ont une définition au niveau du système de la langue, reposant sur la notion d'équivalence sémantique entre formulations, et sur la spécification sémantique et syntaxique des relations entre paraphrases.

Martin (1976 : 77-118) distingue les paires de phrases en relation de paraphrase linguistique (le sens est le même et seuls diffèrent la topicalisation ou les connotations) des paraphrases pragmatiques (« en dépit de différences de sens, elles ont le même sens situationnel » : 118).

Melcuk $(1988)^{\text {iv }}$ : combine des règles d'équivalence lexicales et syntaxiques pour formaliser les relations entre phrases paraphrastiques, ce qui produit un foisonnement de paraphrases possibles, endigué par la mise en évidence de restrictions de sélection, ainsi que par les différenciations que permet une représentation sémantique à trois niveaux: structure sémantique ou invariant de sens, structure sémantico-communicative ou mise en perspective par le locuteur, structure rhétorique spécifiant les buts ou effets visés.

Fuchs (1982) propose une conception énonciative unifiée du fait paraphrastique qui prend ensemble les paraphrases reconnues comme linguistiques et celles qui relèvent de «niveaux référentiel, pragmatique ou symbolique », et elle les analyse du point de vue de l'activité de paraphrasage de la part des locuteurs. Ce qui lui permet ensuite (Fuchs, 1994) de développer une perspective dynamique sur les degrés de parenté sémantique entre énoncés tels qu'ils se trouvent construits par les co-énonciateurs dans des conditions concrètes.

En sémantique générale, Pottier (2000: 117-118) distingue les formulations orthonymiques des nonorthonymiques d'un contenu conceptuel (formulation immédiate, directe vs médiate, issue d'opérations de distanciation, à partir de la notion de représentation juste, prototypique : ortho-). Les phénomènes de métonymie, de métaphore, sont alors inclus dans les relations de parasynonymie ou équivalence sémantique relative entre formulations.

Enfin, nous renvoyons aux travaux de lexicologie appliquée de Picoche (2007), qui fonde une didactique $\mathrm{du}$ vocabulaire sur la reformulation, dont elle propose une typologie pratique (reformulation par changement de l'ordre des mots, par le recours à des dérivés, à des synonymes, ou encore à des paraphrases).

Par ailleurs, lorsque la situation n'est pas monolingue, mais plurilingue, le locuteur peut user de reformulations dans la même langue (intralingues) ou d'une langue à l'autre (interlingues), chacune des 
langues servant d'interprétant à l'autre (Lüdi \& Py, 1986, 2001). Car lorsque plusieurs langues sont en présence, chaque langue peut servir à interpréter l'autre, et le parler bilingue (propre à une communauté bilingue) constitue lui-même un troisième système de communication où les reformulations interlingues peuvent se déployer avec des valeurs spécifiques.

Il y aurait aussi à décliner les types de fonctions de la reformulation, du plus général (fonctions linguistiques) au plus particulier (fonctions dans l'interlocution, puis dans les interactions didactiques). Bornons-nous à inventorier les fonctions pragmatiques (faire varier les effets du message en maintenant l'essentiel du contenu propositionnel), de marquage de point de vue (polyphonie), et les fonctions textuelles de la reformulation au service de la cohésion textuelle (notamment les chaînes anaphoriques), pour ce qui est des situations unilingues.

Dans l'apprentissage d'une / en L2, une reformulation en L2 par le locuteur apprenant d'une L2 constitue une stratégie de formulation lorsqu'il y a manque du mot exact -- le phénomène n'est pas différent de nature de la périphrase décrite chez le locuteur en L1 lorsqu'il y a manque occasionnel du mot, c'est-àdire lorsque le locuteur ne dispose pas d'une solution orthonymique.

Enfin, pour nos situations de classe, la reformulation fonctionne comme un puissant outil pédagogique : dans les échanges entre $\mathrm{M}$ et $\mathrm{EE}$, le $\mathrm{M}$ peut recourir à une nouvelle formulation pour faire passer des contenus, en les rendant plus accessibles (recours à un lexique de base plus fréquent, avec une formulation plus analytique), plus concrets, plus proches de connaissances disponibles. On distingue de ce point de vue des reformulations à fonction d'explication, de clarification, de correction, d'argumentation, ... De leur côté les EE s'approprient les connaissances en les formulant et les reformulant à travers les activités qui leur sont proposées. La reformulation concourt à la fois à la construction des connaissances concernant les objets de savoir, et au développement des connaissances linguistiques des EE, notamment lors des activités en groupe.

\section{Pratiques de classe et usages des reformulations}

Dans cette section, nous examinons les actes de reformulation des maitres et des élèves dans les échanges de classe au primaire, et montrons que ce regard sur les reformulations permet d'éclairer les circuits de construction de connaissances linguistiques et de savoir sur les objets enseignés.

\subsection{Les reformulations dans les séquences de disciplines dites 'non- linguistiques' (DNL)}

Dans cette séquence d'étude du milieu (Education Scientifique et à la Vie pratique selon les programmes du primaire $\mathrm{au}(\mathrm{Togo})^{\mathrm{v}}$, le $\mathrm{M}$ conduit la classe à travers des échanges question-réponse à sa totale initiative vers le but didactique qu'il assigne à cet échange de récapitulation de la leçon antérieure :

(1) M1 la poule + nous avons vu la poule _ n'est-ce pas ? très bien +++ pourquoi élève-t-on la poule ? + pourquoi on élève la poule $?+$ oui $^{\circ}+$

E1 on élève la poule pour sa chair et son oeuf

M2 oui + et pour ses œufs_<écrit au tableau> + pour sa chair + et pour ses oeufs + répète

E2 on élève la poule pour sa chair et ses oeufs

M3 \et pour et pour ses oeufs ++ ses oeufs + n'est-ce pas ? + très bien + de quoi se nourrit la poule ? ++ oui $^{\circ}$ toi

E3 la poule se nourrit des graines

M4 la poule se nourrit de de graines + répète

E4 la poule se nourrit de graines 
M5 la poule se nourrit de graines + nous sommes d'accord ? + c'est tout ? + c'est tout $?+$ oui $^{\circ}$

E5 la poule se nourrit un peu de tout

M6 un peu de tout + c'est vrai + la poule se nourrit un peu de tout_d'abord + la poule se nourrit de graines ${ }^{\circ}+$ à la maison vous laissez la pa/ la poule peut prendre la pâte aussi + c'est vrai $^{\circ}$ non

E6 oui

M7 alors + si la poule se nourrit + un peu de tout + comment nous pouvons l'appeler? ++ toi ?

E7 nous pouvons appeler la poule ${ }^{\circ}+$ un omnivore

M8 un omnivore _ + très bien tu peux épeler un omnivore ${ }^{\circ}+++$ un omnivore $\mathrm{XX}$ monsieur +où est le déterminant faut mettre le déterminant ++ Hadji épèle : un omnivore

E8 un omnivore s'écrit : un U N + omnivore _ O M N N I $\backslash$

M9 \quoi O

E9 O M I V O

M10 $\backslash \mathrm{O} \mathrm{MN}$ I reprends +

E10 omnivore s'écrit : O M N I V O R E

M11 très bien O M N I V O R E _ + c'est un omnivore _ + parce qu'elle c'est ce qu'est la poule parce qu'elle se nourrit un peu de tout $++<\overline{\text { FToEdusivipCM2 }>}$

Les reformulations du $\mathrm{M}$ sont à fonction : a) corrective : M2 rectifie $\mathrm{E} 1$ de façon à inscrire au tableau la phrase exacte à mémoriser, M4 rectifie E3 pour la forme de l'article partitif amalgamé à la préposition de, b) explicative : M6 justifie la formule retrouvée par E5, qui permet d'enchaîner sur l'adjectif technique 'omnivore' dont elle constitue une définition, pour terminer sur la stabilisation orthographique du terme en M8-11 et le rappel du sens associé à 'omnivore'.

Peu après, une autre reformulation permet de rendre de façon analytique le contenu sémantique du verbe technique 'se reproduire' :

(2) M20 comment se reproduit la poule ? +++ comment se reproduit la poule ? ++++ je vous demande est-ce que la poule + pond des oeufs ou bien elle met bas voilà _+ oui $^{\circ}$ non + toi $^{\circ}$

E20 la poule pond des oeufs

M21 la poule pond des oeufs + très bien + la poule pond des oeufs + voilà je vous demande comment se reproduit la poule voilà ${ }^{\circ}$ la question + c'est comme ça que vous allez répondre $<$ FToEdusivipCM2 $>$

Et le même type de reformulation entre un terme technique et sa reformulation analytique conduit ensuite à la stabilisation du terme technique 'ovipare' :

(3) M26 ... un oeuf donne naissance à un petit ${ }^{\circ}+$ poussin + hein c'est clair + la poule pond des oeufs + comment nous pouvons l'appeler? ++

E26 la poule est un ovipare

M27 la poule est un ovipare $c^{\text {‘est-à-dire }}{ }^{\circ}++$ les les les les oiseaux + ou les animaux + qui se reproduisent + par des oeufs + sont des OVIPAres + sommes d'accord + hein ${ }^{\circ}<$ FToEdusivipCM2 $>$

Dans ces classes où les élèves posent rarement des questions, le travail métalinguistique autour des termes apparaît comme un moyen stratégique de clarification des concepts, et aussi de mise en confiance des élèves. Pour s'assurer de l'efficacité de la présentation des mots nouveaux, le maître recourt à des 
procédures métalinguistiques bien rôdées, qui lui permettent de rendre explicites des notions que les élèves éprouveraient des difficultés à saisir hors contexte, dans un environnement scolaire dépourvu de dictionnaire. Les vocables nouveaux sont repris "en mention", en fonction autonymique (cf. AuthierRevuz et coll., 2004), et paraphrasés par une définition. Dans les classes du Togo et particulièrement au primaire, ces procédures métalinguistiques constituent un moyen clé de construction de savoirs sur la langue - objectif qui se substitue bien souvent à la construction de savoirs sur les choses.

Après cette récapitulation, le $\mathrm{M}$ passe à la leçon du jour sur les poissons, qu'il commencera en introduisant le terme 'aquatique' par une paraphrase explicative :

(4) M35 ... nous avons cité des sortes des animaux qui vivent dans l'eau + n'est-ce pas + comment on les appelle + les animaux qui vivent dans l'eau ? comment on les appelle +++++ les animaux qui vivent dans l'eau ++ les animaux qui vivent ${ }^{\circ} /$ oui $^{\circ}$

E35 les animaux qui vivent dans $l^{\prime}$ eau s'appellent ${ }^{\circ}+$ les animaux aquatiques

M36 tRRRès bien + acclamez-le ${ }^{\circ}$

EE <applaudissement rythmés> <FToEdusivipCM2>

Comme on le devine en (2) au tour de parole M21, la visée ultime de la séquence est la préparation des EE aux épreuves, toutes écrites (compositions trimestrielles et examen de fin de cycle primaire), où la connaissance du milieu est évaluée à partir de jeux de questions-réponses écrites brèves, reprises dans des Annales (cf. Noyau, 2007). Cette tendance à la 'littéralité' se reflète directement dans -- ou même est induite par -- les pratiques d'évaluation certificative en vigueur, issues des systèmes scolaires 'classiques' tout français : ainsi, dans les épreuves des disciplines comme la connaissance du milieu, l'histoire ou la géographie, ce qui est requis des élèves est la formulation exacte du résumé, ou une nomenclature, ou une définition, plutôt que des preuves de compréhension, encore moins d'appropriation, des savoirs en question, comme le montrent les études approfondies menées sur les procédures d'évaluation certificative dans plusieurs de ces pays (Noyau, 2006b, 2009).

Cette démarche est entièrement fondée sur la reformulation, c'est-à-dire que la représentation des connaissances est essentiellement intra-linguistique, et très peu mise en relation avec les expériences concrètes des enfants. ${ }^{\text {vi }}$ Elle a souvent pour but d'introduire des termes inconnus pour nommer de façon attendue par la leçon, par le terme technique requis, des réalités bien connues :

(5) $\mathrm{M} \quad$ tout le monde a bien regardé le poisson $+<$ EE oui $>+$ bien ++ le(s) poisson + que nous voyons + le(s) poisson + eh bien le(s) poisson que nous voyons + ils ont quelle forme ? $+\mathrm{i}$ ont quelles formes ? ++ ouais + oui toi $^{\circ}$

E les poissons que nous voyons $\backslash$

$\mathrm{M} \backslash$ le poisson que je vois ${ }^{\circ}$

E le poisson que je vois ${ }^{\circ}+$ a la forme ${ }^{\circ}+$ ovale

$\mathrm{M}$ hein + c'est ovale + ovale très bien + qui va dire autrement ? ++ un autre + regardez celui-ci + regardez ce poisson + il a quelle forme $?+$ regardez +++ oui + regardez ++ eh bien le poisson + regardez il a la forme ${ }^{\circ}+$ al-lon-gée + on dira que il a la forme de ${ }^{\circ}$ fu-seau ${ }^{\circ}+$ hein ${ }^{\circ}$ il est encore un peu allongé comme ça ${ }^{\circ}+$ allongé + donc il a la forme de ${ }^{\circ}+$ fu-seau ${ }^{\circ}++$ est-ce que c'est clair ${ }^{\circ}+<\mathrm{EE} \mathrm{hm}>$ hein ${ }^{\circ}+$ c'est pas tout à fait ovale $^{\circ}+$ mais c'est la forme al-lon-gée ${ }^{\circ}+$ hein ${ }^{\circ}$ allongée + la plupart + des poissons + ont la forme allongée c'tà dire en / de fuseau + allongée (...) la plupart + des poissons + ont la forme allon- $/<$ EE gée $>$ allongée ${ }^{\circ}$ comme celui-ci ${ }^{\circ}$ + voyez $?+$ donc XX on dit le poisson + a la forme + de fu-seau ${ }^{\circ}++$ sommes d'accord $?<$ EE oui $>\quad<$ FToEdusivipCM2 $>$

De même :

(6) $\mathrm{M} \quad$... qui va me donner le nom d'un poisson qui n'a pas le corps couvert d'écailles +++ ouais $\mathrm{B}^{\circ}$

E il y a le silure 
Didactique et enseignement, français langue maternelle, français langue seconde DOI $10.1051 / \mathrm{cmlf} / 2010242$

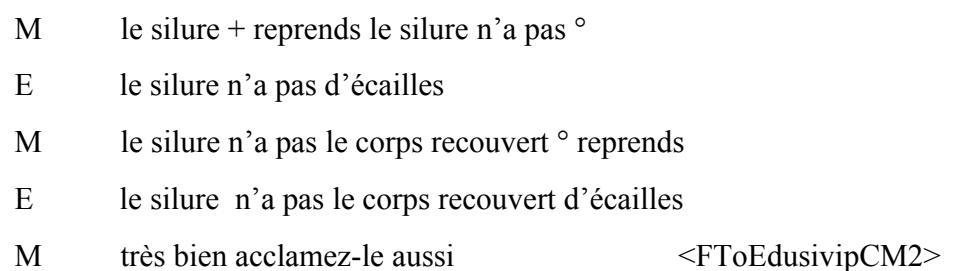

Le $\mathrm{M}$ pratique souvent la sollicitation de reformulations destinée à obtenir des EE l'habitude de s'exprimer par des "phrases complètes" (cf. (6) et (7)) :

(7) $\mathrm{M}$ donc sur le poisson nous avons trois grandes parties + on a la tête ${ }^{\circ}+$ hein ${ }^{\circ}+$

la tête ${ }^{\circ}+$ le $\operatorname{corps}^{\circ}+$ et $^{\circ}+$ quoi + et la queue ${ }^{\circ}+<$ écrit $>$

$\mathrm{M}$ bon + nous sommes d'accord + qui va montrer la tête ? + oui + monsieur + oui +

E je montre la tête

M i faut parler fort

E je montre la tête

$\mathrm{M}$ très bien je montre la tête $+\mathrm{d}$ 'accord montre la tête vous voyez ça là c'est la tête + le corps ${ }^{\circ}+$ où est $^{\circ}$ ça + oui $^{\circ}+$

E je montre le corps

M le corps de quoi ?

E le corps du poisson

$\mathrm{M}$ du poisson _+ très bien _ + et la queue _ montre en même temps la queue ${ }^{\circ}$

E je montre la queue

M la queue de quoi?

E la queue du poisson

$\mathrm{M}$ reprends + je montre ${ }^{\circ}+$

E je montre la queue du poisson

$\mathrm{M}$ je montre la queue $\mathrm{du}^{\circ}+$ poisson $+\quad<$ FToEdusivipCM2 $>$

Dans l'exemple suivant tiré d'une leçon sur les vases communicants au CM2, le M produit une suite de reformulations de la notion clé de physique 'vases communicants' à partir du sens prototypique de chacun des mots qui la dénomment : la bifocalisation se résout, ici encore, au détriment de la construction de la notion de physique, en se référant seulement à des équivalences en langue. On peut penser que cette façon de présenter la notion permettra aux EE d'en mémoriser la formulation, mais difficilement de comprendre la nature du phénomène physique dont il est question. Si les questions d'examen sur ce thème se bornent à des définitions ou sollicitations de la dénomination exacte, le but quant à la préparation aux épreuves sera peut-être atteint ...

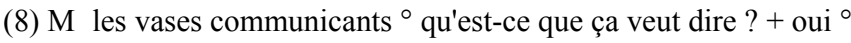

$\mathrm{M}+\mathrm{EE}<\ldots>$

$\mathrm{M} \quad$ bon ++ tout ce que vous dites là vous ne répondez pas à ma question $+++\mathrm{je}$ dis ${ }^{\circ}$ qu'est-ce que vous entendez par vase ? puits ? les vases communicants ? celui qui m'a dit un pot + un vase + un pot $^{\circ}$ un vase $^{\circ}$ ça peut aller mais pas de jet +++ on dit communicant qui se communique $+\mathrm{c}^{\prime}$ est comme je suis en train de vous parler et vous me répondez +++ nous sommes en train de nous communiquer + on peut se communiquer par des gestes $+++\mathrm{ici}^{\circ}$ lorsque nous mettons de l'eau dans des vases on dit que ces vases se communiquent c'est-à-dire que l'eau peut partir de ce vase pour se retrouver dans l'autre vase et dans les deux vases nous aurons notre eau qui peut se 
retrouver au même niveau + dans le premier comme dans le deuxième +++ et en ce moment comme les personnes se communiquent par la parole ${ }^{\circ}$ eh bien les objets qui retiennent l'eau et que l'eau se retrouve au même niveau + on sous-entend pour dire que les vases là se communiquent +++ c'est comme ils se parlent +++ $<$ FToScienCM2>

\subsection{Les reformulations dans les séquences de travail de la langue}

Il est fréquent, tant en classe que dans les interactions ordinaires en français des adultes scolarisés, que le locuteur propose deux formulations alternatives présentées parallèlement dans l'énoncé, le choix revenant au récepteur dans les interactions ordinaires. Mais la fonction remplie change en contexte didactique, où les EE sont invités à enrichir leur répertoire lexical de signifiants sans diversifier les sens associés :

(9) $\mathrm{M}$ Bien nous allons tout à l'heure écouter une histoire $+\underline{\text { un récit }+ \text { ou bien un }}$ $\underline{\text { conte }}$ sur les animaux + sauvages + nous sommes d'accord ${ }^{\circ}$

EE oui <FToLgeCM1Hyene\&Renard $>$

Dans le travail de la langue, le M sollicite souvent des formulations successives des EE, pour atteindre par approximations la formulation visée, à l'aide de la formule rituelle « qui va dire mieux ? », comme ici dans une tâche de restitution de texte après lecture : vii

(10) M11 qu'est-ce qui a permis à Ozi de gagner ? (...) oui ${ }^{\circ}$ Fayisa

E11 Ozi a sauté pour gagner

M12 Ozi a sauté pour gagner_qui va dire mieux ? votre camarade dit que bon Ozi a sauté pour gagner Kodjo _ c'est Kodjo qu'on a gagné hein +++ vous me levez les doigts _ comment ? oui ${ }^{\circ}$ hein oui ${ }^{\circ}$

E12 Ozi a sauté de côté

M Ozi a sauté de côté ${ }^{\circ}$ pour pouvoir + vaincre son $^{\circ}+$ son son prochain _ donc il a sauté de côté et + d'un seul coup il l'a + terrassé _ + bien <FToElocCE2 $>$

Dans la même leçon d'Elocution à partir d'un extrait littéraire vu antérieurement, avec comme objectif central l'extension du vocabulaire, se déroule un rituel pédagogique constitué de trois phases réitérées appliquées à chaque vocable : $a$ ) sélection par les EE d'un mot nécessitant élucidation ; $b$ ) demande aux EE d'en donner une définition, rejetée ou acceptée sans commentaires qualitatifs, suivie d'une définition type fournie par le $\mathrm{M}(11) ; c$ ) demande aux EE de construire des phrases isolées formellement acceptables contenant ce vocable, que ceux-ci écrivent sur leur ardoise et oralisent sur demande du M, en s'appuyant sur la définition donnée, avec validation par acceptation ou rejet sans commentaire. L'élucidation des conditions d'emploi du vocable se fera donc exclusivement à base de reformulations successives par les EE, refusées ou acceptées. Là il n'est plus question de reformulation, mais de phrases acceptables ou non en tant que telles, autour d'une notion prégnante et non problématisée de 'phrase correcte'.

(11) M17 oui + ces jeux se passent la nuit au clair de lune + devant une grande foule venue de tous les villages environnants + oui ${ }^{\circ}$ qui peut expliquer $?+$ de tous les villages environnants ++ tous les villages environnants ++ ça ne vous dit rien ?++ cela se passe de tous les villages environnants ++ oui $\mathrm{A}^{\circ}$

E17 cela se passe de tous côtés

M18 de tous côtés + très bien + qui va dire mieux + ça se passe de tous côtés c'est bien + qui va dire mieux ++ oui $^{\circ}$

E18 cela se passe autour

M19 cela se passe autour + oui + qui va dire mieux + oui ${ }^{\circ}$

E19 les villageois de de de tous ce villages sont venus à cette lutte 


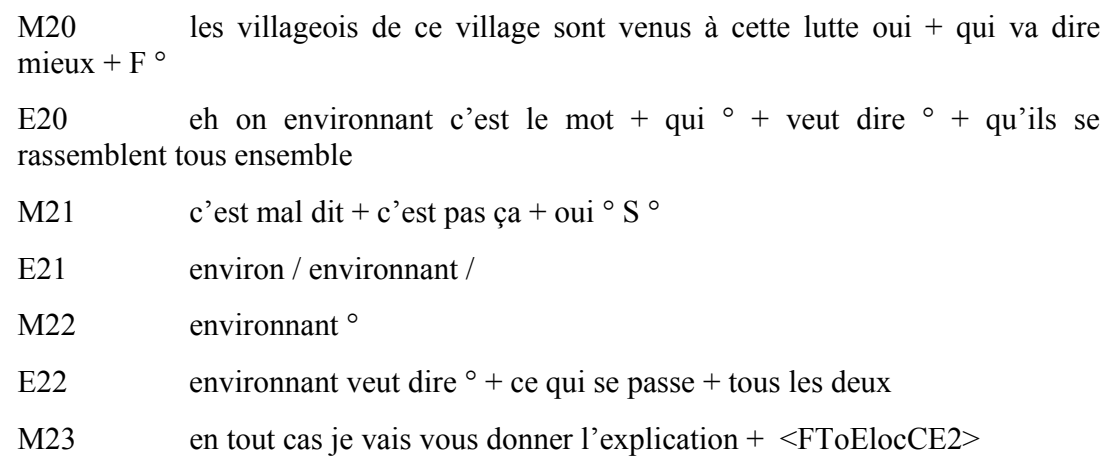

\subsection{Passages entre l'oral et l'écrit et reformulation}

Dans le travail de classe, les reformulations successives d'un objet de savoir en construction passent de l'oral à l'écrit et de l'écrit à l'oral en fonction des activités proposées (cf. Garcia-Debanc et Volteau, 2007). Nous avons analysé, dans la perspective d'évaluer les apprentissages proposés, permis, ou bloqués ches les EE par le travail de formulation des maîtres, une séance de travail oral (Elocution) sur un conte traditionnel, au CM1 : La hyène et le renard ${ }^{\text {viii }}$ qui est particulièrement favorable au déploiement de reformulations. Le $\mathrm{M}$ est un enseignant chevronné directeur d'école, et à ce titre représentatif des pratiques d'enseignement en vigueur, qu'il a à charge de transmettre aux enseignants nouvellement recrutés. Il part d'un dessin des animaux protagonistes qu'il a tracé au tableau avant la séance (en s'inspirant de près de l'illustration du manuel où il a puisé ce conte) et, après les avoir fait identifier par les $\mathrm{EE}$, lit le conte, inclus dans le manuel de lecture d'une année antérieure, puis en donne une version orale, «à ma manière ", où sont détaillés avec l'aide du tableau les parcours des protagonistes au fil de l'histoire.

Puis il demande directement aux EE une restitution de cette version oralisée. Une demi-douzaine d'EE « redisent» en monologue ce qu'ils ont retenu de l'histoire. Enfin, le M propose à la classe des questions de compréhension sur le conte, pour associer aux échanges les autres EE, et clôture la séquence par des échanges correctifs à partir d'un énoncé d'E sur l'accord sujet-verbe.

Pour contribuer à notre propos sur le rôle central des reformulations dans l'appropriation de la langue et des savoirs, nous synthétisons ici l'analyse des reformulations présentées dans ce corpus : celles du texte écrit dans la version orale du $\mathrm{M}$, les reformulations dans les restitutions du conte par quelques EE après la phase d'élucidation, ainsi que celles d'autres EE en dialogue sur sollicitation du M. ${ }^{\text {ix }}$

\subsubsection{Les reformulations du maître dans l'oralisation d'un texte}

D'abord, la comparaison entre la version imprimée du texte et sa lecture initiale à la classe (TE $\rightarrow$ TL) révèle une lecture scrupuleusement exacte quant à la formulation, et qui marque par la prosodie de façon nette et différenciée les différentes segmentations de la ponctuation. Cette exactitude permet la stabilisation en mémoire de traces textuelles bien structurées chez les EE qui ont peu de contacts avec l'imprimé.

Nous considérons donc essentiellement les formulations consécutives à cette première version orale reflétant le texte imprimé. D'abord une version plus libre du $\mathrm{M}$, construite dans l'interaction avec la classe, que nous appelons le texte oralisé (TO). Le M annonce la couleur : il s'agit d'abord d'assurer la bonne compréhension du conte :

(12) TO : M Voilà l'histoire + je vais reprendre à ma manière + pour que vous puissiez bien comprendre + il s'agit de deux amis + hein ${ }^{\circ}$ ici c'est l'hyène (il montre le tableau) vous voyez ici c'est l'hyène + et là le renard _ + c'est dans le monde animal + et en ce temps là + c'est le temps de famine + le temps de disette où y avait pas à manger ${ }_{-}+$hein ${ }^{\circ}$... 
Les variations du TO par rapport au conte écrit concourent à différentes fonctions :

a) adaptation au registre oral, i. par des énoncés de pure cohésion (ex. « et qu'est-ce qui s'est passé? », « en ce temps-là quand il a écouté ceci que fait le renard? »), alors que des tournures du récit lettré ne sont pas reprises (ex. «TL : «l'hyène prend aussitôt la parole et dit», «à ces paroles »), ii. par des choix syntaxiques diminuant le taux de condensation ${ }^{\mathrm{x}}$ (ex. TL : pensant être le seul à avoir vu le gibier, TO : et elle fait semblant de n'avoir pas vu la biche morte), et iii. par des décompositions analytiques du sens :

(13) TL : chacun prend alors un chemin différent / TO : bien + le renard aussi a pris ce chemin (...) + l'hyène aussi a pris ce chemin + + (= cet autre chemin, montré au tableau sur le schéma d'un carrefour)

b) élucidations lexicales, par simple variation synonymique vers un élément plus courant, ou par paraphrase explicative à fonction définitoire, les deux étant éventuellement combinés :

(14) TL : C'était au temps d'une grande famine / TO : M c'est le temps de

famine + le temps de disette où y avait pas à manger

c) explicitations d'inférences, explications sur les liens causaux entre événements et états de choses ou sur les buts des protagonistes (ex. les deux se promènent pour voir s'il peuvent trouver quelque chose ; comme le renard aussi veut la viande, ...), concrétisations :

(15) TL : chacun d'eux (...) ne dit rien à son compagnon _+ / TO : comme le renard $\underline{\text { aussi veut la viande }}^{\circ}+$ il n'a rien dit à son amie l'hyène $+\underline{l^{\prime} \text { hyène aussi a vu }}$ d'avance cette ${ }^{\circ}$ biche morte + donc elle veut la manger seule elle n'a rien dit à son ami le renard $t^{\mathrm{xi}}$

d) aide à la mémorisation par des reformulations multiples de certains segments, avant de demander aux EE de restituer le conte :

(16) TL cette biche n'est pas à partager _ + elle appartiendra au plus âgé de nous deux

+ / TO : l'animal + la viande là donc ${ }^{\circ}$ va revenir à celui qui ${ }^{\circ}$ est le plus grand +

$\bar{l}$ 'animal reviendra au plus âgé c'est à dire au plus ${ }^{\circ}$ grand ++1 'hyène dit + la

viande là n'est pas à partager _ + hein ${ }^{\circ}$ donc ${ }^{\circ}$ elle revient au plus âgé +

On comprend aisément les vertus de ces reformulations en cascade pour assurer la compréhension et la mise en mémoire du conte par les EE. Certaines de ces reformulations voient confirmée leur efficacité par la reprise ou reformulation des contenus associés dans toutes les versions des EE qui suivront (cf. 3.3.2 infra).

Inversement, plusieurs énoncés du TL ne sont pas repris dans le TO, on peut s'expliquer que le M les ait négligées parce que ce sont des inférences de liaison (Denhière \& Baudet, 1991), qui se trouvent explicitées dans la version rédigée du conte destinée à la lecture enfantine mais peuvent aisément être récupérées lors de la compréhension. Ces énoncés présents dans le seul TL se trouvent disparaitre de toutes les versions des EE (ex. : ils dépassent la bête morte ; l'hyène prise de pitié se tourne vers son ami ...)

Ces reformulations du $\mathrm{M}$ sont très rarement signalées par des marqueurs spécifiques, mais simplement juxtaposées : la construction et l'ajustement du sens se font par approximations voir accumulations, sans souci de différencier les conditions d'usage des différentes formulations.

Rappelons par ailleurs que les reformulations relevées dans l'interprétation orale du texte par le $\mathrm{M}$ manifestent aussi les spécificités du lecte de ce $\mathrm{M}$ par rapport à la langue normée, ce lecte servant de cible à l'acquisition de la langue par les EE (cf. Noyau 2000, 2006a ; Peuvergne 2005).

\subsubsection{Les reformulations du conte par les élèves}

Outre le TL et le TO du M, nous disposons de trois versions complètes du conte par des EE immédiatement après l'écoute du TO, plusieurs autres EE s'y étant essayés mais pour quelques fragments seulement. Les énoncés qui sont présents dans toutes ces versions (TL, TO, E2, E3, E6+7) constituent en quelque sorte l'ossature du conte - mais lacunaire, insuffisante pour capter le scénario dans son entier. Nous verrons qu'en effet certaines versions des EE reprennent des parties mémorisées, mais sans pouvoir 
reconstituer le scénario : ils n'ont compris l'histoire que partiellement. Or il s'agit d'un conte reposant sur la prise de pouvoir par la ruse, type le plus fréquent des contes africains et qui n'est pas sans rappeler la veine du Roman de Renart. Ce type, du point de vue textuel, entraîne le recours massif au discours rapporté, car les armes principales de la ruse sont les paroles. Une de ces paroles clé oubliée, et on manque la stratégie des protagonistes. Une de ces paroles clé déplacée, et on manifeste une compréhension défectueuse du scénario. Les segments qui trouvent des équivalents dans toutes les versions sont les suivants :

(17) l'hyène et le renard se promenaient - ils trouvent ... une biche morte - chacun d'eux pensant être le seul à avoir vu le gibier ne dit rien à son compagnon - nos deux amis se retrouvent ... auprès du cadavre - l'hyène ... dit - cette biche n'est pas à partager - puis elle continue - moi je suis née avec le monde - et lui dit $<$ au renard $>$ ne pleure pas - alors le renard répond - ... mon fils ...

Les dernières paroles rapportées, la réplique du renard qui dame le pion à l'hyène en matière d'ainesse, et qui constituent la pointe de l'histoire (c'est que ta date de naissance me rappelle un triste souvenir, en effet c'est à cette époque que mon premier fils est mort), sont reprises par tous sauf par E2 qui mentionne vaguement « l'histoire de mon fils » : il n'a donc pas compris ce qui fait cette histoire.

La reprise à l'identique par les EE d'un segment du TL modifié dans le TO, donc après une seule écoute, se produit dans un seul cas : l'énoncé de la situation initiale, ce segment verbal étant entouré de formulations variées des arguments du verbe :

(18) TL un jour l'hyène et le renard se promenaient ensemble / TO donc les deux se promènent / E2 un jour l'hyène et le renard se promenaient dans leur ( $\mathrm{xx}$ / E3 les deux se promenaient et en parlant + les deux se promenaient en famine / E6+7 l'hyène et le renard se promenaient dans la brousse

Dans le TL l'ensemble de l'exposition de la situation initiale est à l'imparfait, puis le conte se déroule au présent de narration ; dans le TO seule une proposition de l'exposition maintient l'imparfait (« temps de disette où y avait pas à manger »), tout le reste étant narré au présent et au passé composé, avec d'autres temps dans les paroles rapportées (futur périphrastique, futur simple) comme il est naturel. Dans les reformulations des EE, la narration est surtout au passé composé + imparfait + plus-que-parfait à valeur de passé composé, conformément à leur entraînement à la narration au passé qui est au programme. Ils font un usage des temps plus varié que le $\mathrm{M}$, qui a sans doute voulu faciliter la tâche de la restitution du conte par les EE - et que le texte. ${ }^{\text {xii }}$

De quels types linguistiques de reformulation, selon les catégories proposées par Picoche (2007), fait usage le $\mathrm{M}$ dans ses versions successives et reprises en dialogue du conte ? Il s'agit là de reformulations auto-initiées, donc toutes compatibles avec le répertoire linguistique disponible, et qui répondent à des fonctions communicatives et pragmatiques entrainées par la situation didactique spécifique.

a) une seule reformulation par changement de l'ordre des mots (entraînant un changement de la relation thème - rhème)

(19) M Oui il ne pleure pas à cause de la biche + il pleure à cause de quoi ${ }^{\circ}+q u$ 'estce qui le fait pleurer ${ }^{\circ}$ oui ${ }^{\circ}$

Le fait de reformuler la question sous une autre forme élargit les possibilités pour les EE de proposer une réponse, dans cette activité conçue pour stimuler l'expression orale.

b) toute une gamme de reformulations au moyen de synonymes, jouant sur différents degrés de précision (nous disons de granularité sémique : cf. Noyau \& Paprocka, 2001), ou variant les points de vue sur l'objet. Ainsi, à propos de la biche convoitée :

(20) TL biche morte - bête morte - cadavre - biche - viande / TO biche qui est morte - la viande - biche morte - l'animal - la biche - cadavre - l'animal la viande là - cette biche / E2 la biche (x 4) / E3 la biche (x 3) - la biche morte (x 2) - cette biche / E6+7 la biche morte (x 2) - la biche (x 7) / M en dialogue : une biche morte - la biche - cette biche 
On voit que TL et TO déclinent toute une gamme de points de vue sur la bête, alors que les EE se bornent à 'biche' (+/- morte). Pour ce qui est de la désignation des actes de parole, c'est le TL seul qui varie, alors que le $\mathrm{M}$ et les EE s'en tiennent au verbe de base 'dire' :

(21) TL dit - trouvent une raison pour - prend la parole et dit - continue - à ces paroles - répond / TO n'a rien dit - dit / EE dit - ne veut pas dire - a dit

c) au moyen de paraphrases (définies par Picoche comme variation libre sur la forme et les contenus du texte source, en y incluant la métaphore)

(22) TL famine / TO temps de disette où y avait pas à manger (cf. (14) supra)

Parmi les nombreuses reformulations du M de TL dans TO pour faciliter la compréhension, une bonne partie consiste en reformulations par décomposition analytique de notions complexes, comme pour 'carrefour', que le M explique via un schéma au tableau au fil de TO :

(23) $\mathrm{M}$ bien + à un moment donné + quand ils arrivent + quand ils arrivent + à un carrefour + carrefour voilà c'est un chemin ${ }^{\circ}+$ de cette manière ${ }^{\circ}+$ de cette façon ++ alors ${ }^{\circ}$ ils ont trouvé une biche qui est un animal + sauvage également + qui est morte ici +

Mais le $\mathrm{M}$ ne recourt pas à des reformulations au moyen de dérivés (fondées sur la relation $\mathrm{N}$ - Adj ou sur la relation $\mathrm{V}-\mathrm{N}$ ), ni au moyen de synonymes jouant sur le niveau de langue. On voit par là que cette activité n'est pas tournée ves un objectif d'aprentissage lexical.

Les reformulations sont particulièrement riches en ce qui concerne la question clé pour ce conte de l'âge et du droit d'ainesse, vue par l'hyène qui fixe l'attribution du gibier selon le droit d'ainesse et assène la preuve verbale de son antériorité (Hy), puis par le renard (Ren) qui retourne la situation à son profit. On trouve alors notamment des reformulations du M par des synonymes exprimant des points de vue différents sur la réalité :

(24) TL (Hy) elle appartiendra au plus âgé de nous deux ... je suis née avec le monde donc je suis la plus âgée ... tu es bien jeune ... quand tu seras vieux ... (Ren) ta date de naissance me rappelle un triste souvenir + en effet c'est à cette époque que mon premier fils est mort / TO $(\mathrm{Hy})$ va revenir à celui qui est le plus grand + l'animal reviendra à au plus âgé c'est-à-dire au plus grand ... donc elle revient au plus âgé.. je suis née avec le commencement du monde ... tu n'es pas encore grand toi aussi tu seras vieux et tu auras des biches (Ren) ce que tu m'as dit me rappelle la date où mon premier fils mon premier enfant est mort ... quand tu m'as dit cette date-là je me souviens que c'est à cette date où mon premier fils est mort / E2 (Hy) moi je suis né moi j'ai né au début du monde (Ren) moi je raconte l'histoire de mon fils $<$ E2 n'a pas compris $>$ / E3 (Hy) c'est l'homme c'est la personne qui est le plus grand qui va manger cette biche ... je suis née avec / au commencement du monde (Ren) quand tu as dit cette date j'ai pensé à la date que mon fils était mort / E6+7 (Hy) ça revient pour la personne qui est grande .. moi j'ai né à la commencement du monde (Ren) la date que l'hyène a dit me rappelle la mort de mon première fils + mon première fils était mort à la commencement du monde

Les adjectifs âgé, grand et vieux semblent recouvrir le même sens (l'ainesse au sens strict, notion clé dans l'organisation sociale endoculturelle) et s'opposent ensemble à jeune. D'ailleurs, en français togolais, une grande personne désigne ordinairement une personne âgée et/ou respectée (cf. en éwé ame-gan 'personne + grand' $=$ id.).

Cette notion-clé du conte prend encore diverses autres formes dans le dialogue $\mathrm{M}-\mathrm{EE}$ qui suit, où le $\mathrm{M}$ explicite de façon exhaustive les inférences relatives à l'âge que recèle le dénouement du conte, et que les reformulations de certains $\mathrm{EE}$ ont révélé comme pas toujours comprises :

(25) s'il faut croire à ce que dit le renard _ et ce que dit l'hyène ${ }^{\circ}+$ quel $^{\circ}$ animal est le plus âgé? _ quel animal est le plus grand _ + d'après ce que disent les deux $+{ }_{+}^{+}$ alors qui est le plus grand (...) 


\section{E c'est l'hyène}

M pourquoi ${ }^{\circ}(\ldots)$

E le renard a accouché un enfant

$\mathrm{M}$ à cette date déjà + lui ${ }^{\circ}+$ il a accouché + hein ${ }^{\circ}$ l'hyène dit que lui là est né avec le monde ${ }_{-}+$hein ${ }^{\circ} c^{\prime}$ t-à dire au commencement ${ }_{-}+$en ce temps déjà comment? _ je suis grand je suis marié ${ }^{\circ} \mathrm{j}^{\prime}$ ai déjà / mon premier fils est mort à cette date $^{\circ}+$ on a vu? $^{\circ}+$ donc $^{\circ}$ à écouter les deux ${ }^{\circ}$ le renard devient l'animal le plus âgé ${ }_{-}+$et qui finalement va manger cette biche ${ }^{\circ}+$ qui va finalement manger la biche?

E c'est le renard

$\mathrm{M}$ c'est le renard ${ }_{-}+$hein ${ }^{\circ}+$ bien ${ }_{-}+$nous continuons ${ }_{-}+$chacun maintenant a compris le texte

Et quels types linguistiques de reformulation trouve-t-on chez les EE qui reprennent le conte après l'écoute des deux versions TL et TO?

Chez un sujet seulement (E6), on trouve une reformulation par changement de l'ordre des mots, qui entraîne le recours à des noms déverbaux non utilisés dans TL ni TO : la mort, la perte, signe d'une compétence linguistique plus développée que la moyenne du groupe :

(26) TL c'est à cette époque que mon premier fils est mort / TO la date où mon premier fils (...) est mort / E3 la date que mon fils était mort / E6 la mort de mon première fils (...) mon première fils était mort à la commencement du monde (...) la perte de ma première fils

Le type le plus représenté chez les EE est la reformulation au moyen de synonymes avec diminution du degré de précision (granularité sémique moindre), comme le recours aux verbes de base : tous les actes de parole sont désignés par dire. Cette tendance est logiquement à mettre en relation avec la limitation du répertoire lexical des apprenants. Ce qui explique également l'absence de reformulations par des synonymes jouant sur le niveau de langue, ou exprimant des points de vue différents sur la réalité. Enfin, pour ce qui est des reformulations par paraphrases (variation libre sur la forme et les contenus du texte source), on trouve de deux types : a) paraphrase de décomposition analytique du sens :

(27) TL carrefour / TO carrefour voilà c'est un chemin de cette manière (croquis au tableau) / E6 le chemin est divisé en deux

(28) TL nos deux amis se retrouvent nez à nez auprès du cadavre / TO le renard + il a pris ce chemin ${ }^{\circ}$ a contourné _ vers l'animal _ + l'hyène aussi ${ }^{\circ}$ contourne + et il vient ici _ + voilà les deux ${ }^{\circ}+\operatorname{les}^{-}$deux se retrouvent ${ }^{\circ}+$ E6 à un moment +1 'hyène aussi reveni / revenut chez la / chez la biche ${ }^{\circ}+$ le renard aussi reveni chez la biche

Dans (28), le $\mathrm{M}$ avait déjà adopté une décomposition analytique du sens, adaptée à la capacité linguistique des EE, mais avec un verbe spatial rare, les EE eux adoptent cette stratégie, mais avec un verbe plus courant.

b) paraphrases ajoutant une modalisation, selon point de vue des protagonistes :

(29) TO il n'a rien dit / E2 il ne veut pas dire ça à le renard E3 il ne veut pas dire à le renard

(30) TL + TO ne pleure pas / E2 Il ne faut pas pleurer E6 faut pas pleurer

Les verbes modaux vouloir et falloir sont déjà acquis, et permettent à certains EE de reformuler des énoncés portant sur des actes de communication en leur ajoutant une orientation subjective intradiégétique. 


\subsubsection{Les reformulations dans la sollicitation des élèves par des échanges question- réponse}

Enfin, quelles reformulations des EE favorise le $\mathrm{M}$ dans le dialogue sur le conte ? Les sollicitations des élèves par des questions permettent à la fois de faire reprendre l'énoncé d'un $\mathrm{E}$ par un autre, avec correction mutuelle entre EE, d'améliorer la forme en reprenant la parole d'un E, et de solliciter des commentaires explicatifs. Il s'agit de reformulations hétéro-initiées déclenchées par le guidage pédagogique du M. Ainsi, voici comment le début du même dialogue final est sollicité et traité dans la phase de questions-réponses :

(31) M Est-ce que l' hyène a dit comment partager la biche? ${ }^{\circ} \quad(\cdots)$

E l'hyène a dit + il faut pas pleure

$\mathrm{M}$ non + + on ne dit pas il faut pas pleure + il ne faut pas pleurer $+\mathrm{Et}^{\circ}$

E L'hyène a dit celui qui est grand il va prendre ce mange

M Qui va reprendre la phrase là?

$\mathrm{E}$ L'hyène $\mathrm{a} \mathrm{dit}^{\circ}+$ celui qui est grand c'est celui qui va manger la biche +

M L' hyène a dit celui qui est grand + hein $+c$ 'est lui ${ }^{\circ}+$ qui va manger la biche +

hein + en ce temps là le petit lui ne mangeait pas + lui aussi sera grand + et il

trouvera aussi + sa proie -+ on a compris? +

Mais le $\mathrm{M}$ effectue surtout des reformulations à fonction corrective, concernant notamment la détermination des $\mathrm{SN}$, ou des expressions plus ou moins inappropriées (cf. aussi début de (31)) :

(32) E ils ont trouvé la biche / M ils ont trouvé une biche morte

(33) $\mathrm{E}$ ils se sont séparé la route / M ils ont pris le chemin différent ... chacun a repris son chemin

ou bien il sollicite de la classe des formulations plus proches du texte source :

(34) $\mathrm{M}$ après avoir écouté l'hyène + qu'est-ce que le renard a fait ? + oui ${ }^{\circ}$

E le renard a pleuré

$\mathrm{M}$ le renard $?^{\circ}+$ oui $?^{\circ}+$

$\mathrm{E}^{\prime}$ le renard commence à pleurer

$\mathrm{M}$ le renard commence ${ }^{\circ}+$ hein $^{\circ}+$ à pleurer _ + bien

dans une visée de convergence vers le TL source.

Les catégories linguistiques de reformulation proposées par Picoche visent avant tout, rappelons-le, à favoriser un apprentissage lexical intégré par des manipulations textuelles et des familles de reformulations, de façon à créer chez les apprenants des réseaux ou 'grappes' lexicales (cf. Picoche \& Rolland, 2002 ; Chevalier \& Noyau, 2008) conduisant aisément à la mise en texte. La visée de la séquence étudiée n'est pas centrée sur le lexique, c'est pourquoi sans doute certaines catégories n'y sont pas ou très peu représentées : variation de l'ordre des éléments avec changement de diathèse, synonymie fondée sur la dérivation, ou avec variation du niveau de langue, paraphrase par métaphore, alors que d'autres sont privilégiées : reformulations multiples par synonymie, paraphrases par décomposition analytique du sens. La visée d'entraînement à l'expression orale a déterminé un certain profil de reformulations.

\subsubsection{Bilan des reformulations dans une séquence de langue orale ('Elocution')}

Il est clair que les séquences d' «Elocution», qui visent à «faire parler» les EE selon l'expression 
favorite des maîtres, se prêtent au jeu de la reformulation davantage que les séquences des disciplines tenant à la connaissance du milieu (Histoire, Géographie, Sciences d'observation), car l'exactitude des formulations n'a pas ici d'enjeu évaluatif. Ce qui fait enjeu, c'est malgré tout encore et toujours la bonne forme phrastique, que le M sollicite entre EE par des demandes de corrections mutuelles, et en même temps le commentaire du contenu, qui est censé conférer du sens aux phrases et les relier à un contexte d'expérience. L'abondance des reformulations n'est pas utilisée de façon stratégique pour permettre aux $\mathrm{EE}$ de rendre leur répertoire linguistique plus précis ou nuancé. Il semblerait que l'abondance en ellemême suffit, sans visée structurante se souciant du sens, comme le montrait déjà la séquence d'élocution commentée en (11). C'est là un aspect auquel les $\mathrm{M}$ semblent accorder peu d'attention, et qui par ailleurs est peu pris en considération dans les procédures évaluatives, les MM eux-mêmes ayant développé leur attention à la langue et leur souci correctif et évaluatif essentiellement dans deux directions : la grammaire phrastique, et l'orthographe. On pourrait d'ailleurs avancer que ce flou dans la compétence sémantique, associé à une faible conscience métalinguistique des sens lexicaux, mis en évidence dans la façon dont sont conduites ces séquences d'Elocution, constitue l'une des causes de la valorisation irrestrictive du respect de la lettre. Et que le domaine sémantique devrait constituer un objectif de formation initiale et continue des enseignants utilement intégré à un travail sur les reformulations.

\section{Pour la formation des maîtres aux usages de la reformulation}

\section{$4.1 \quad$ Objectifs}

A partir des analyses de pratiques de classe dont les sections précédentes donnent un aperçu, il s'agira de proposer des contenus pour des actions de formation des maîtres, visant à : a) renforcer leur maitrise de la langue française, dont ils sont locuteurs de référence pour les élèves ; b) modifier leur conception rigide de la langue normée dans le sens d'une conception plus dynamique de la langue en action; c) les convaincre de l'efficacité pédagogique de la reformulation chez le $\mathrm{M}$ et les $\mathrm{EE}$ et les pourvoir de méthodes pour la faire pratiquer. Car il est important, nous l'avons vu, que les MM acceptent des EE plusieurs formulations, et encouragent et valorisent le fait d'essayer de dire avec des moyens détournés en l'absence de / ou lors de manque de l'orthonyme (Pottier, 2000), et sachent structurer ces moyens de dire autour des orthonymes, et en hiérarchisant les choix lexicaux en fonction des relations d'hyperonymie et co-hyponymie.

Ainsi, la formule omniprésente « qui va dire mieux ? » : quelle incitation est-ce réellement ? Et comment aménager les activités didactiques au profit d'un usage de la langue à la fois plus précis et plus sensible aux contextes, permettant de conférer un rôle pilote au sens? C'est le fil conducteur de l'outil en chantier dont le $\S$ suivant présente les principes.

\subsection{Un outil de formation multimédia fondé sur des observations de pratiques de classe}

Nous avons choisi le DVD comme outil d'un dispositif de formation ciblée, permettant un travail systématique d'analyse et de réflexion proactive sur la pratique enseignante, à partit d'échantillons concrets des pratiques de classe dans les écoles, et conduisant à l'évolution de ces pratiques (Noyau, à paraître).

L'ensemble pédagogique prévu comprend :

a) une série de montages de séquences de classe enregistrées en vidéo : chaque montage, d'une durée approximative de 8 à $10 \mathrm{~min}$, où les principales phases de la séquence sont identifiées par des intertitres, comprend des moments caractéristiques successifs d'une activité ou leçon d'une durée de $30 \mathrm{~min}$. à une heure.

b) un livret d'accompagnement, comportant une présentation (objectifs de la formation, démarches utilisées et notions en cause), une fiche d'exploitation par séquence avec transcription détaillée des 
passages pertinents, et des outils de récapitulation et d'auto-évaluation.

Le support sera décliné pour des utilisations en présentiel et à distance avec autoformation, c'est-à-dire que le même matériel peut entrer dans plusieurs dispositifs de formation, compte tenu des besoins massifs en perfectionnement pédagogique dans le contexte considéré. Il s'agit actuellement d'un prototype à expérimenter sur le terrain.

\subsection{Esquisse de la démarche proposée}

Pour chacun des montages de séquences vidéo, il s'agira de mettre en évidence les reformulations effectuées et leurs fonctions, les occasions de reformulation non saisies ou à aménager. Les activités proposées associeront des phases de prise de conscience, problématisation, travail sur la langue, et production d'outils didactiques.

Le travail sur la langue est conçu dans une démarche positive d'enrichissement du lexique par la mise en réseau des unités lexicales via les reformulations, de l'ordre du perfectionnement linguistique, qui est à bien distinguer de la remédiation (dont ces maîtres ont parfois besoin). Il alliera des techniques d'enrichissement associant la prise de conscience des ressources linguistiques et des conditions de leur usage à la pratique de gammes de formulations, en vue d'un accroissement de la flexibilité contextuelle et de la capacité expressive, et d'une maîtrise accrue de la reformulation à fonction didactique.

\section{Références bibliographiques}

Allal L., Ch. Anane, Sénémaud M.\& C. Noyau (2002). Construction des énoncés et connecteurs dans la structuration des récits enfantins en arabe tunisien et en français. LINX 46 'Les connecteurs', 133-151.

Authier-Revuz J., Doury M. \& S. Reboul-Touré, eds. (2004). Parler des mots : le fait autonymique en discours. Paris, Presses de la Sorbonne Nouvelle.

Bange P. (1992). A propos de la communication et de l'apprentissage en L2, notamment dans ses formes institutionnelles, A.I.L.E, 1, Nouvelles perspectives dans l'étude de l'apprentissage d'une langue étrangère en milieu scolaire et en milieu social, 53-85.

Boré, C. (2000). Le brouillon, un introuvable objet d'étude ? Pratiques $n^{\circ} 105-106,23-49$.

Bretegnier, A. \& G. Ledegen (2002). Sécurité / insécurité linguistique. Hommage à Nicole Gueunier. Paris : L'Harmattan, 'espaces francophones' / Université de La Réunion.

Chevalier G. \& C. Noyau (2008). Le perfectionnement du vocabulaire chez les étudiants acadiens de niveau universitaire. Communication au Congrès de la FIPF, Québec, juillet.

Denhière G. \& S. Baudet (1991). Lecture, compréhension de texte et science cognitive. Paris : Presses Universitaires de France.

Elalouf M.-L. (éd.) (2005). Ecrire entre 10 et 14 ans. Un corpus, des analyses, des repères pour la formation, Versailles, :CRDP, 2005.

De Gaulmyn, M.-M. 1987. Actes de reformulation et processus de reformulation. in L'analyse des interactions verbales - la dame de Caluire: une consultation., P. Bange, éd. Berne: Peter Lang, 'sciences pour la communication', 83-98.

Fuchs, C. (1994). Paraphrase et énonciation. Gap / Paris : Ophrys.

Fuchs, C. (1982). La paraphrase. Paris : PUF.

Gajo, L. (2007). Linguistic knowledge and subject knowledge: How does bilingualism contribute to subject development? The International Journal of Bilingual Education and Bilingualism, Vol. 10, No. 5.

Garcia-Debanc C., Volteau S. (2007). Formes linguistiques et fonctions des reformulations dans les interactions scolaires, Recherches linguistiques 29, 'Usages et analyses de la reformulation'. Metz : Université de Metz, 309- 
340.

Garry, R.-Ph. ; Karsenti, Th. ; Benziane, A. et Baudot, F. (2008). Former les enseignants du XXIème dans toute la francophonie, à travers quatre thèmes : le français, vecteur d'interculturalité ; un Master francophone de formation des enseignants ; les TIC, outils de complémentarité pour la formation initiale et continue ; la formation des maîtres et l'éducation pour tous. (Actes du 2e Colloque du RIFEFF). Agence Universitaire de la Francophonie (AUF) / Réseau International Francophone des Etablissements de Formation de Formateurs (RIFEFF), $379 \mathrm{p}$.

Gülich, E. \& T. Kotschi (1987). Les actes de reformulation dans la consultation La dame de Caluire. in L'analyse des interactions verbales - la dame de Caluire: une consultation, P. Bange (éd.). Berne : Peter Lang, 'sciences pour la communication', 15-81.

Karsenti, T. ; Garry, R.-P. ; Béchoux, J. ; et Tchameni Ngamo, S., eds. (2007). La formation des enseignants dans la francophonie : diversités, défis et stratégies d'action. (Actes du 1er Colloque du RIFEFF). Montréal : AUF, 388 p.

Kihlstedt, M. (2005). Stratégies compensatoires dans l'acquisition du lexique verbal chez des élèves en immersion scolaire en L2 français, In F. Grossmann, G. Petit, M.-A. Paveau, E. Calaque, J. David (eds) Apprentissage du lexique : langue, cognition, discours. Grenoble : ELLUG, 85-105.

Lüdi, G. \& B. Py (1986, 2001). Etre bilingue. Berne : Peter Lang.

Lyster, R. \& Izquierdo, J. (2009). Prompts versus recasts in dyadic interaction. Language Learning, 59 (2), 453-498.

Lyster, R. (2002). Negotiation in immersion teacher-student interaction. International Journal of Educational Research, 37, 237-253.

Martin, R. (1976). Inférence, antonymie et paraphrase. Paris : Klicksieck.

Martinot C. (2000). Etude comparative des processus de reformulation chez des enfants de 5 à 11 ans. Langages 140, 2000, Acquisition et reformulation, 92-123.

Martinot C. \& A. Ibrahim, eds. (2003). La reformulation, un principe universel d'acquisition, Paris : Kimè.

Melc'uk, Igor (1988). Paraphrase et lexique dans la théorie Sens-Texte. Lexique 6, 'Lexique et paraphrase', 13-54.

Milicevic, Jasmina (2007). La paraphrase. Modélisation de la paraphrase langagière. Berne : Peter Lang, 'Sciences pour la communication'.

Noyau C. (2001). Le français de référence dans l'enseignement du français et en français au Togo. In: M. FRANCARD, G. GERON \& R. WILMET (dir.). 2000-2001. Le français de référence. Constructions et appropriations d'un concept. Actes du colloque de Louvain-la-Neuve (3-5 novembre 1999). Volume II : Cahiers de l'Institut de Linguistique de Louvain, 27 (1-2), 57-73.

Noyau C. (2002a). Construire les savoirs scolaires via la scolarisation en français langue seconde en Afrique : quelle littéracie viser, et par quelles voies? Communication au Colloque 'La littéracie : le rôle de l'école', IUFM de Grenoble, 24-26 octobre.

Noyau C. (2002b). Les choix de formulation dans la représentation textuelle d'événements complexes : gammes de récits. Journal de la Recherche Scientifique de l'Université de Lomé (Togo), Vol. 2/2002, 33-44.

Noyau C. (2003). Apprentissages disciplinaires et langagiers dans la scolarisation en français langue seconde: repérages dans le temps en histoire au primaire. in Construction des connaissances et langage dans les disciplines d'enseignement (Actes sur CD-Rom), J.-P. Bernié, M. Jaubert et M. Rebière, éds. CIRFEM-DAEST, Université Victor Segalen-Bordeaux II, $16 \mathrm{pp}$

Noyau C. (2004). Appropriation de la langue et construction des connaissances dans l'école de base en pays francophone : du diagnostic aux actions. In : Penser la francophonie. Concepts, actions et outils linguistiques. Actes des Premières Journées scientifiques communes des réseaux de chercheurs concernant la langue (Ouagadougou, Burkina Faso, 31 mai - 2 juin 2004). Paris: AUF / Eds des Archives Contemporaines, coll. 'actualité scientifique', 473-486.

Noyau C. (2005). Le lexique verbal dans l'acquisition d'une langue seconde : verbes de base, flexibilité sémantique, granularité. In F. Grossmann, M.-A. Paveau, G. Petit, eds. Apprentissage du lexique: langue, cognition, discours. Grenoble, ELLUG, 65-84. 
Noyau C. (2006a). Le langage des maîtres comme français de référence : rôle de l'école dans la transmission de la langue. Le Français en Afrique, 21. 339-350.

Noyau C. (2006b). Linguistique acquisitionnelle et intervention sur les apprentissages : appropriation de la langue seconde et évaluation des connaissances à l'école de base en situation diglossique. Bulletin VALS/ASLA 83/1 'Les enjeux sociaux de la linguistique appliquée', Neuchâtel (Suisse), 93-106.

Noyau C. (2007). L'enseignement de disciplines non linguistiques en FLS au primaire en Afrique de l'ouest : les sciences d'observation à travers les manuels et les activités de classe. TREMA 28, septembre, Carlo, A. \& Verdelhan-Bourgade, M. (dir.), Montpellier, IUFM, 49-62.

Noyau C. (2008). Place des verbes dans le Français Fondamental, acquisition du lexique verbal en français langue seconde, et didactique du lexique. in R. Bouchard \& C. Cortier (eds.) Pratiques et représentations de l'oral en FLES, 50 ans après le français fondamental. Le Français dans le Monde - Recherches et applications, $\mathrm{n}^{\circ}$ spécial, janvier, 87-100.

Noyau C. (2009). Modalités d'optimisation du passage de L1 à L2 dans l'enseignement primaire en contexte multilingue. Mali, Mauritanie, Seychelles. Paris : OIF / Le Web Pédagogique Eds., 305 p.

Noyau C. (à paraître). Passages entre L1 et L2 en classe : dispositif de formation à partir de séquences de classe pour les formateurs de l'école bilingue. in : Former à distance des formateurs : stratégies et mutualisation dans la francophonie, Actes du IIIe Colloque du RIFEFF (Niamey novembre 2009).

Peuvergne J. (2005). Etude de glissements syntaxiques et sémantiques dans le parler d'enseignants togolais de français à Lomé. in K. Ploog \& B. Rui (eds.) Situations de plurilinguisme et enseignement du français en Afrique, 99-115.

Picoche J. (2007). La reformulation, base de l'enseignement du vocabulaire. Recherches linguistiques 29, 'Usages et analyses de la reformulation', M. Kara (ed.), 293-308.

Picoche, J. \& Rolland, J.-C. (2002). Dictionnaire du français usuel : 15000 mots utiles en 442 articles (+ cd-rom). Bruxelles : De Boeck, Duculot.

Pottier B. (2000). Représentations mentales et catégorisations linguistiques. Louvain / Paris : Peeters. (§ 21a, b, i)

Viberg A. (2002). Basic verbs in second language acquisition. Revue Française de Linguistique Appliquée 2002/2 'Acquisition des langues: tendances récentes', 51-69.

Volteau S. \& Garcia-Debanc C. (2008). Les reformulations de l'enseignant dans quatre situatios scolaires : existe-t-il une influence de l'objet enseigné sur les types de reformulations utilisées ? in M. Schuwer, M.-C. Le Bot \& E. Richard, eds. Pragmatique de la reformulation. Types de discours, interactions didactiques. Rennes : Presses Universitaires de Rennes, 253-268.

Volteau S. \& Garcia-Debanc C. (2009). Gérer les reformulations : un geste professionnel. Influence des objets enseignés sur les types de reformulations. in Bucheton D., Dezutter O. eds. Le développement des gestes professionnels dans l'enseignement du français. Un défi pour la recherche et la formation, Bruxelles : De Boeck, 191-212.

i Abréviations utilisées : $\mathrm{E}$ : élève ; EE : élèves ; $\mathrm{M}$ : maître ; MM :maîtres ; L1 : langue première ; L2 : langue seconde ou ultérieure ; DNL : discipline non linguistique.

ii Sur cette notion, voir Viberg (2002); Noyau (2005a).

iii Tâche proposée en vue d'études acquisitionnelles comparatives par Martinot (2000). Cf. Martinot \& Ibrahim, eds. (2003).

iv Développé par Milicevic (2007).

v Conventions de transcription : E : élève; EE : élèves; $\mathrm{M}$ : maître ; +,,+++++ : pause courte / longue ; nnnnn : séquence inaudible; (et alors) : séquence incertaine; alors ${ }^{\circ}$ : intonation montante ; alors : intonation descendante; $\mathrm{xxx} /$ : auto-interruption; $\mathrm{xxx} \backslash:$ hétéro-interruption; $\mathrm{XXXXxxxx}$ : syllabe(s) à accentuation emphatique $;(\ldots)$ : fragment supprimé dans une intervention; $<\ldots>$ : tour(s) de parole supprimé(s).

vi Qui sont chargés de nourrir les poules à la maison, même en ville, et assistent au quotidien à la 
transformation des poissons, base de l'alimentation, en nourriture.

vii Notons le caractère neutre - voire ambigu - de la formule, qui ne délivre aucun retour négatif, ni positif. Aux EE de deviner si leur proposition pouvait être acceptable - quoique non orthonymique - ou non. Seule une formulation sera saluée comme satisfaisant les attentes, et récompensée par des applaudissements, après quoi on passe à autre chose.

viii Enregistrement effectué par Muriel Cordier dans le cadre de la pré-enquête du projet CAMPUS / AUF / ACI Cognitique «Appropriation du français via la scolarisation en situation diglossique » (2002-2005), dans une école primaire de zone rurale à $30 \mathrm{~km}$ de la capitale du Togo, l'enseignant de la classe étant le directeur de l'école.

ix Nous adoptons les raccourcis suivants pour désigner les différentes formulations successives : TE $=$ texte écrit $; \mathrm{TL}=$ texte lu $; \mathrm{TO}=$ texte oralisé $; \mathrm{TR}=$ texte restitué (élève). Là où c'est utile, les formulations modifiées sont en italiques, les ajouts sont soulignés.

x La condensation est estimée par le nombre de prédications par énoncé et le degré de hiérarchisation hypotactique (cf. Allal et coll. 2002 ; Noyau et coll. 2005).

xi En français du Togo, l'adverbe de cohésion aussi indique qu'on traite deux actants dans des prédications mises en parallèle, mais n'implique pas que quoi que ce soit dans la prédication $a$ soit mis en facteur commun : « Nous sommes trois maîtres dans l'école. Un prend CP1-CP2, moi CE1-CE2 et l'autre aussi CM1-CM2. » (maître d'école communautaire)

On laissera pour une autre étude l'usage des temps verbaux par ces élèves. 